\title{
Superconductivity above 200K Observed in Superhydrides of Calcium
}

\section{Zhiwen Li}

Chinese Academy of Sciences

Xin He

Chinese Academy of Sciences

Changling Zhang

Chinese Academy of Sciences

\section{Sijia Zhang}

Chinese Academy of Sciences

Yating Jia

Institute of Physics, Chinese Academy of Sciences

\section{Shaomin Feng}

Institute of Physics, Chinese Academy of Sciences

$\mathrm{Ke} \mathrm{Lu}$

Chinese Academy of Sciences

\section{Xiancheng WANG}

Institute of Physics

Jianfa Zhao

Institute of Physics, Chinese Academy of Sciences

\section{Youwen Long}

Institute of Physics https://orcid.org/0000-0002-8587-7818

\section{Richeng Yu}

Institute of Physics, Chinese Academy of Sciences

\section{Luhong Wang}

Harbin Institute of Technology

\section{Meiyan Ye}

Zhanshuo Zhang

Jilin University

Vitali Prakapenka

University of Chicago

\section{Stella Chariton}

The University of Chicago

Haozhe Liu

Center for High Pressure Science and Technology Advanced Research 
Changqing Jin ( $\sim$ Jin@iphy.ac.cn )

Chinese Academy of Sciences https://orcid.org/0000-0001-8097-9156

\section{Article}

Keywords:

Posted Date: December 9th, 2021

DOI: https://doi.org/10.21203/rs.3.rs-1126263/v1

License: (c) (1) This work is licensed under a Creative Commons Attribution 4.0 International License. Read Full License

Version of Record: A version of this preprint was published at Nature Communications on May 23rd, 2022. See the published version at https://doi.org/10.1038/s41467-022-30454-w. 


\section{Superconductivity above $200 \mathrm{~K}$ Observed in Superhydrides of Calcium}

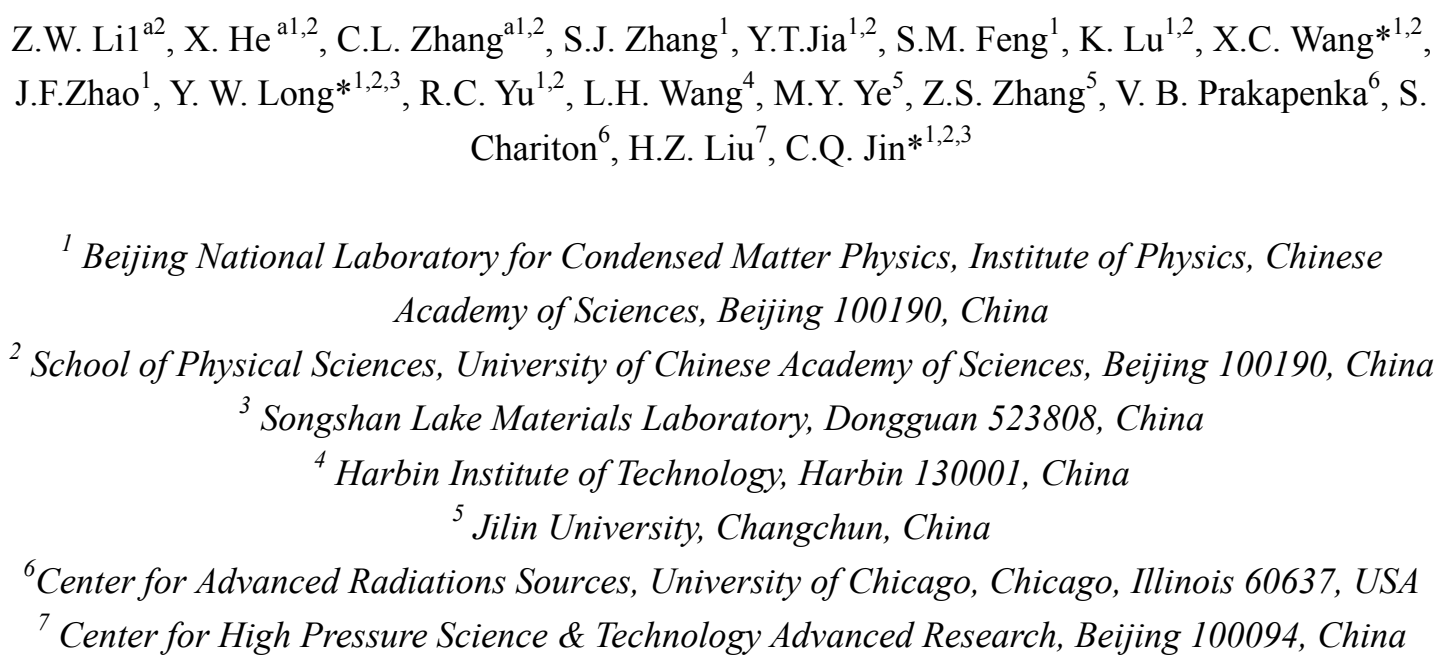

Searching for superconductivity with Tc near room temperature is of great interest both for fundamental science \& potential applications. Here we report the experimental discovery of superconductivity with maximum critical temperature(Tc) above $210 \mathrm{~K}$ in calcium superhydrides, the third type hydride experimentally showing superconductivity above $200 \mathrm{~K}$ in addition to sulfur hydride \& rare earth hydride system. The materials are synthesized at the synergetic conditions of $160 \sim 190 \mathrm{GPa}$ and $\sim 2000 \mathrm{~K}$ using diamond anvil cell combined with in-situ laser heating technique. The superconductivity was studied through in situ high pressure resistance measurements in applied magnetic field for the sample quenched from high temperature while maintained at the synthesized pressure. The upper critical field was estimated to be $\sim 268 \mathrm{~T}$ while the GL coherent length is $\sim 11$ A. The in situ $x$ ray diffractions with synchrotron suggest that the synthesized calcium hydrides are primarily composed of $\mathrm{CaH}_{6}$ while there also exist other calcium hydrids with different hydrogen.

a:Contributed Equally

*The Corresponding Authors: Wangxiancheng@iphy.ac.cn; Jin@iphy.ac.cn 


\section{Introduction}

The superhydrides received growing attentions recently because of the high temperature superconductivity ${ }^{[1]}$. It was suggested that superhydrides are likely to be a step toward metallic hydrogen since the "chemically precompressed" hydrogen in the superhydrides can decrease the critical pressure required for the metallization ${ }^{[1-3]}$. Because of high phonon frequencies and strong electron phonon coupling, high temperature superconductivity was suggested in hydrogen rich materials. Sulfur hydride is one of examples, in which the high Tc superconductivity was reported both theoretically \& experimentally at Megbar level pressures ${ }^{[4,5]}$. In addition, alkali earth, rare earth and other superhydrides are theoretically suggested to possess high temperature superconductivity at high pressures ${ }^{[6-10]}$. Those metal superhydrides favor to form hydrogen rich coordination such as clathrate like cage structures at high pressure where the metal ions are located in the center of the $\mathrm{H}$ clathrats and act as carrier donor. The occupation of the unfilled anti-bonding $\sigma^{*}$ orbitals of the $\mathrm{H}_{2}$ molecules by the electrons from the metal ions would enhance to dissociate the $\mathrm{H}_{2}$ molecules hence to form $\mathrm{H}$ clathrats. Recently rare earth Lanthanum (or Yttrium) hydrides are also experimentally reported to be superconductive with Tc exceeds 200 $\mathrm{K}^{[12-16]}$. However, there are no experimental observations for superconductivity above $200 \mathrm{~K}$ so far in alkaline earth superhydrides, in spite of the superconductivity with Tc $\sim 20 \mathrm{~K}$ was reported in barium hydrides ${ }^{[17]}$. Here, we report the experimental realization of high Tc superconductivity in calcium superhydrides synthesized at high pressure and high temperature conditions. The superconductivity with Tc $210 \mathrm{~K}$ at $160 \sim 190 \mathrm{GPa}$ was observed. Hence in addition to sulfur hydrides, rare earth hydrides this is the third type of superconducting hydrides with Tc above $200 \mathrm{~K}$.. 


\section{Experiment}

The diamond anvil cell techniques are used for both synthesis \& measurements of the calcium hydrides. The diamond anvils are of optical pure quality with culet diameter of $100 \mu \mathrm{m}$ beveled to $300 \mu \mathrm{m}$ for the experiments. The electrodes for resistance measurements are embedded in the sample chamber before high pressure high temperature synthesis. The experiments are conducted in two steps: (I) synthesis of the calcium hydrides at high pressure high temperature; (II) the follow up measurements of superconductivity for the sample quenched from high temperature but remained at the same pressure. The T301 stainless steel served as gaskets that are prepressed from $250 \mu \mathrm{m}$ to $\sim 20 \mu \mathrm{m}$ in thickness at about $20 \mathrm{GPa}$. A hole of $300 \mu \mathrm{m}$ in diameter was drilled at the center of imprint. The hole filled with fine insulating cubic boron nitride $(\mathrm{cBN})$ powder mixed with epoxy that was pressed with anvils to form a solid layer of $\sim 15 \mu \mathrm{m}$ in thickness. A hole of $70 \mu \mathrm{m}$ in diameter was drilled at the center of the solid cBN layer to serve as the sample chamber. The Pt foils with the thickness of $0.5 \mu \mathrm{m}$ were deposited on the surface of the culet as the inner electrodes. The gold wire was attached to the Pt foil to serve as the outside electrodes. The calcium specimen with a shape of $30 \mu \mathrm{m}$ in width $* 30 \mu \mathrm{m}$ in length and $2 \mu \mathrm{m}$ in thickness was stacked on the inner electrodes. A flake of ammonia borane filled into the sample chamber plays the role of hydrogen source (hydrodizer) while it also the pressure transmitting medium ${ }^{[14]}$. The sample loading as well as electrodes performance are conducted in the glove box filled with flowing Ar gas with 1ppm oxygen or water trace to avoid moisture or contamination. The setups are followed the ATHENA procedure reported Ref 18.

The sample was first applied to the pressure of 160 190 GPa followed by heating 
at $\sim 2000 \mathrm{~K}$ for $\sim 30 \mathrm{~min}$. The heating was performed with a pulse laser beam of $1064 \mathrm{~nm}$ wave length. The spot of laser beam is about $5 \mu \mathrm{m}$ in diameter with power of $20 \mathrm{~W}$. The heating temperature was estimated by fitting the black body irradiation spectra. The heated sample was quenched from high temperature while maintained at high pressure. A piston cylinder type anvil cell composed of $\mathrm{BeCu}$ was adopted in the experiments. The synthesized sample compressed within diamond anvil cell was put into a MagLab system to perform the electric conductivity measurements using a Van der Pauw method with $1 \mathrm{~mA}$ applied current. The MagLab system can provide synergetic extreme environments with temperatures from $300 \mathrm{~K}$ to $1.5 \mathrm{~K}$ and a magnetic field up to $9 \mathrm{~T}^{[19]}$. Pressure was calibrated by the shift of the first order Raman edge frequency from the cutlet of diamond ${ }^{[20,21]}$.

The synchrotron x-ray diffraction experiments in the diamond anvil cell were carried out at GSECARS of the Advanced Photon Source at the Argonne National Laboratory. The x-ray with $\lambda=0.3344 \AA$ was focused down to $\sim 3$ um diameter spot on the sample. The symmetric diamond anvil cell was used to generate pressure with bevel diamond anvils (100/300um ) and rhenium gasket indented to $25 \mu \mathrm{m}$. Samples were loaded into the pressure chamber without the insulated layer of $\mathrm{cBN}$ and $\mathrm{Pt}$ electrodes that were used for resistive measurements. Calcium hydrides was synthesized in situ at $175 \mathrm{GPa}$ with laser heating at $2000 \mathrm{~K}$. The sample pressure in these synchrotron $\mathrm{X}$ ray diffraction experiments has been determined by both the shift of Raman edge frequency from the cutlet of diamond and the equation of state for rhenium. The X-ray diffraction images are converted to two dimensional diffraction data with Dioptas ${ }^{20}$. 


\section{Results \& Discussions}

Fig. 1(a) displays the image of the sample assembly. The dotted red square is the sample shape and the four gray dotted regions are the inner electrodes. Fig. 1(b) shows the measurements of resistance as function of temperature at $160 \mathrm{GPa}$ for sample A. The resistance curves almost coincide for both the cooling and warming cycles. The resistance drops at around $210 \mathrm{~K}$ and approaches to zero at low temperature suggesting that the superconducting like transitions take place in the calcium superhydrides. Fig. 2 shows the resistance measurements by application of magnetic fields for sample A. It is seen that the whole transition curves shift to low temperature with the critical temperature gradually suppressed by the applied field, in consistent with the nature of superconductivity. The inset of Fig. 1(b) displays the enlarge view of the derivative of resistance over temperature around the transition region, from which the $\mathrm{Tc}^{\text {onset }}$ of $210 \mathrm{~K}$ is estimated at right side upturn point. In addition to the first transition at $\sim 210 \mathrm{~K}$, there are also several drop kinks at $\sim 180 \mathrm{~K}$ and $\sim 160 \mathrm{~K}$ as shown in Fig.1(b). These drop kinks imply multistep superconducting transitions that are supported by the broaden transition curves shown in Fig.2. In the context of calculation, the calcium hydrides such as $\mathrm{CaH}_{6}, \mathrm{CaH}_{10}, \mathrm{CaH}_{12}$ etc are stable or metastable at high pressure and show high Tc superconductivity ${ }^{[2,6,11]}$. Hence it is likely that these multistep transitions should be attributed to calcium hydrides with different hydrogen amount, which are probably synthesized due to inhomogeneity of either pressure or hydrogen. It is natural that there exists large pressure gradient in pressure cell at megabar pressure, while the hydrogen inhomogeneity likely arises from the inhomogeneous laser heating to ammonia borane. We synthesized couples of samples to optimize the single transition quality either by reducing sample size in 
order to improve pressure homogeneity or to heat sample in a more homogeneous scanning manner. Fig. 3(a) presents the resistance as function of pressures measured at $185 \mathrm{GPa}$ for samples $\mathrm{B}$ and $\mathrm{C}$, where one step sharp with $\mathrm{Tc} \sim 200 \mathrm{~K}$ aresachieved. The superconductivity is supported by the measurements at applied magnetic field as shown in Fig. 3(b).

To estimate the upper critical field $H_{c 2}(0)$, the superconductivity as function of magnetic field was investigated based on sample $\mathrm{C}$. The Tc values are determined with the criteria of the $\mathrm{Tc}^{\text {onset }}$, the temperature at $90 \%$ and $50 \%$ of normal state resistance, respectively. By linear fitting the $H_{c 2}(T)$, the slope of $d H_{c 2} /\left.d T\right|_{T c}$ was obtained to be $-1.976 \mathrm{~T} / \mathrm{K}$ for the $\mathrm{Tc}^{\text {onset }},-1.726 \mathrm{~T} / \mathrm{K}$ for the $90 \%$ of normal state resistance and $-1.389 \mathrm{~T} / \mathrm{K}$ for the $50 \%$ of normal state resistance, respectively. Using the Werthamer-Helfand-Hohenberg (WHH) formula of $\mu_{0} H_{c 2}(T)=-0.69 \times$ $d H_{c 2} /\left.d T\right|_{T c} \times T_{c}$, the $H_{c 2}(0)$ value was calculated to be $268 \mathrm{~T}, 227 \mathrm{~T}$ and $179 \mathrm{~T}$ for the criteria of $\mathrm{Tc}^{\text {onset }}$, the $90 \%$ and the $50 \%$ of normal state resistance, respectively. The Ginzburg Landau (GL) formula of $\mu_{0} H_{c 2}(T)=\mu_{0} H_{c 2}(0)\left(1-\left(T / T_{c}\right)^{2}\right)$ is also used to estimate the upper critical field at zero temperature. As shown in Fig. 4 the fitting of the $\mu_{0} H_{\mathrm{c} 2}(\mathrm{~T})$ by GL formula gives a value of $H_{c 2}(0)$ ranging from $131 \mathrm{~T}$ to $195 \mathrm{~T}$ using criteria of $\mathrm{Tc}^{\text {onset }}$, the temperature at $90 \%$ and $50 \%$ of normal state resistance, respectively. They are comparable to those reported in $\mathrm{LaH}_{10}{ }^{[12]}$. Using the obtained value of $H_{c 2}(0)=131 \sim 268 \mathrm{~T}$, one can roughly estimate the GL coherence length $\xi$ to be $11 \sim 16 \AA$ via the equation of $\mu_{0} H_{c 2}(0)=\Phi_{0} / 2 \pi \xi^{2}$, where $\Phi_{0}=2.067 \times 10^{-15}$ Web is the magnetic flux quantum.

The possible superconducting phases are investigated by high pressure x-ray diffraction experiments and it was suggested that the primary phase of the 
superconducting sample is composed of $\operatorname{Im} \overline{3} m$ phase of $\mathrm{CaH}_{6}$ as shown in Fig. S1-3. We became aware during preparing the paper that an independent work by Ma et al. was carried with the similar results ${ }^{[23]}$.

\section{References:}

[1]. N. W. Ashcroft, "Hydrogen dominant metallic alloys: High temperature superconductors?". Phys. Rev. Lett. 92, 187002 (2004).

[2]. D. V. Semenok, I. A. Kruglov, I. A. Savkin, A. G. Kvashnin, A. R. Oganov, "On Distribution of Superconductivity in Metal Hydrides". Curr Opin Solid St M 24, 100808 (2020).

[3]. J. A. Xu, Z. W. Zhu, "Metallic Hydrogen". Physics 6, 296 (1977).

[4]. D. F. Duan, Y. X. Liu, F. B. Tian, D. Li, X. L. Huang, Z. L. Zhao, H. Y. Yu, B. B. Liu, W. J. Tian, T. Cui, "Pressure-induced metallization of dense (H2S)(2)H-2 with high-T-c superconductivity". Sci. Rep. 4, 6968 (2014).

[5]. A. P. Drozdov, M. I. Eremets, I. A. Troyan, V. Ksenofontov, S. I. Shylin, "Conventional superconductivity at 203 kelvin at high pressures in the sulfur hydride system". Nature 525, 73 (2015).

[6]. H. Wang, J. S. Tse, K. Tanaka, T. Iitaka, Y. M. Ma, "Superconductive sodalite-like clathrate calcium hydride at high pressures". Proc Natl Acad Sci U S A/ PNAS 109, 6463 (2012).

[7]. F. Peng, Y. Sun, C. J. Pickard, R. J. Needs, Q. Wu, Y. M. Ma, "Hydrogen Clathrate Structures in Rare Earth Hydrides at High Pressures: Possible Route to Room-Temperature Superconductivity". Phys. Rev. Lett. 119, 107001 (2017).

[8]. H. Y. Liu, I. I. Naumov, R. Hoffmann, N. W. Ashcroft, R. J. Hemley, "Potential high-T-c superconducting lanthanum and yttrium hydrides at high pressure". Proc Natl Acad Sci U S A/ PNAS 114, 6990 (2017).

[9]. P. P. Kong, V. S. Minkov, M. A. Kuzovnikov, A. P. Drozdov, S. P. Besedin, S. Mozaffari, L. Balicas, F. F. Balakirev, V. B. Prakapenka, S. Chariton, D. A. Knyazev, E. Greenberg, M. I. Eremets, "Superconductivity up to $243 \mathrm{~K}$ in the yttrium-hydrogen system under high pressure". Nat. Commun. 12,5075 (2021).

[10]. J. A. Flores-Livas, L. Boeri, A. Sanna, G. Profeta, R. Arita, M. Eremets, "A perspective on conventional high-temperature superconductors at high pressure: Methods and materials". Phys. Rep. 856, 1 (2020).

[11]. Z. J. Shao, D. F. Duan, Y. B. Ma, H. Y. Yu, H. Song, H. Xie, D. Li, F. B. Tian, B. B. Liu, T. Cui, "Unique Phase Diagram and Superconductivity of Calcium Hydrides at High Pressures". Inorg. Chem. 58,2558 (2019).

[12]. A. P. Drozdov, P. P. Kong, V. S. Minkov, S. P. Besedin, M. A. Kuzovnikov, S. Mozaffari, L. Balicas, F. F. Balakirev, D. E. Graf, V. B. Prakapenka, E. Greenberg, D. A. Knyazev, M. Tkacz, M. I. Eremets, "Superconductivity at $250 \mathrm{~K}$ in lanthanum hydride under high pressures". Nature 569, 528 (2019).

[13]. Z. M. Geballe, H. Y. Liu, A. K. Mishra, M. Ahart, M. Somayazulu, Y. Meng, M. Baldini, R. J. Hemley, "Synthesis and Stability of Lanthanum Superhydrides". Angew. Chem. Int. Edit 57, 688 (2018).

[14]. M. Somayazulu, M. Ahart, A. K. Mishra, Z. M. Geballe, M. Baldini, Y. Meng, V. V. Struzhkin, R. J. Hemley, "Evidence for Superconductivity above $260 \mathrm{~K}$ in Lanthanum Superhydride at Megabar 
Pressures". Phys. Rev. Lett. 122, 027001 (2019).

[15]. F. Hong, L. X. Yang, P. F. Shan, P. T. Yang, Z. Y. Liu, J. P. Sun, Y. Y. Yin, X. H. Yu, J. G. Cheng, Z. X. Zhao, "Superconductivity of Lanthanum Superhydride Investigated Using the Standard Four-Probe Configuration under High Pressures". Chin. Phys. Lett. 37, 107401 (2020).

[16]. E. Snider, N. Dasenbrock-Gammon, R. Mcbride, X. Wang, N. Meyers, K. V. Lawler, E. Zurek, A. Salamat, R. P. Dias, "Synthesis of Yttrium Superhydride Superconductor with a Transition Temperature up to 262 K by Catalytic Hydrogenation at High Pressures". Phys. Rev. Lett. 126, 117003 (2021).

[17]. W. Chen, D. V. Semenok, A. G. Kvashnin, X. Huang, I. A. Kruglov, M. Galasso, H. Song, D. Duan, A. F. Goncharov, V. B. Prakapenka, A. R. Oganov, T. Cui, "Synthesis of molecular metallic barium superhydride: pseudocubic BaH12". Nat. Commun. 12, 273 (2021).

[18]. Y. T. Jia, X. He, S. M. Feng, S. J. Zhang, C. L. Zhang, C. W. Ren, X. C. Wang, C. Q. Jin, "A Combinatory Package for Diamond Anvil Cell Experiments". Crystals 10, 1116 (2020).

[19]. J. L. Zhang, S. J. Zhang, H. M. Weng, W. Zhang, L. X. Yang, Q. Q. Liu, S. M. Feng, X. C. Wang, R. C. Yu, L. Z. Cao, L. Wang, W. G. Yang, H. Z. Liu, W. Y. Zhao, S. C. Zhang, X. Dai, Z. Fang, C. Q. Jin, "Pressure-induced superconductivity in topological parent compound $\mathrm{Bi}_{2} \mathrm{Te}_{3}$ ". Proc Natl Acad Sci U S A/ PNAS 108, 24 (2011).

[20]. S. J. Zhang, X. C. Wang, R. Sammynaiken, J. S. Tse, L. X. Yang, Z. Li, Q. Q. Liu, S. Desgreniers, Y. Yao, H. Z. Liu, C. Q. Jin, "Effect of pressure on the iron arsenide superconductor $\mathrm{Li}_{\mathrm{X}} \mathrm{FeAs}$ (x=0.8,1.0,1.1)". Phys. Rev. B 80, 014506 (2009).

[21]. H. K. Mao, J. Xu, P. M. Bell, "Calibration of the Ruby Pressure Gauge to 800-Kbar under Quasi-Hydrostatic Conditions". Journal of Geophysical Research-Solid Earth and Planets 91, 4673 (1986).

[22]. C. Prescher, V. B. Prakapenka, "DIOPTAS: a program for reduction of two-dimensional X-ray diffraction data and data exploration". High Press. Res. 35, 223 (2015).

[23]. L. Ma, K. Wang, Y. Xie, X. Yang, Y.-Y. Wang, M. Zhou, H.-Y. Liu, G.-T. Liu, H.-B. Wang, Y.-M. Ma, "Experimental observation of superconductivity at $215 \mathrm{~K}$ in calcium superhydride under high pressures". arXiv:2103.16282 (2021). 


\section{Acknowledgments:}

The work is supported by NSF, MOST \& CAS of China through research projects. Portions of this work were performed at GeoSoilEnviroCARS (The University of Chicago, Sector 13), Advanced Photon Source (APS), Argonne National Laboratory. GeoSoilEnviroCARS is supported by the National Science Foundation - Earth Sciences (EAR - 1634415) and Department of Energy- GeoSciences (DE-FG02-94ER14466). This research used resources of the Advanced Photon Source, a U.S. Department of Energy (DOE) Office of Science User Facility operated for the DOE Office of Science by Argonne National Laboratory under Contract No. DE-AC02-06CH11357.We are grateful to Prof. J. G. Cheng, J.P.Hu \& L. Yu for the discussions. We thank Prof. T. Xiang, B. G. Shen, \& Z. X. Zhao for the consistent encouragements. 


\section{Author Contributions}

Research Design \& Supervision: C.Q.J.; high pressure synthesis and in situ resistance measurements: Z.W. L., X.H., C.L.Z., S.J.Z., Y.T.J., S.M.F., Y.W.L.,X.C.W., R.C.Y, J.F.Z., C.Q.J; in situ synchrotron experiments: L. H. W., M.Y.Y., Z.S.Z., V.B.P., S.C., H.Z.L., manuscript writing: Z.W.L., X.C.W. and C.Q.J. All authors contributed to the discussions.

Competing interests: The authors declare no competing interests.as defined by Nature Portfolio, or other interests that might be perceived to influence the results and/or discussion reported in this paper. 


\section{Figure Captions:}

Fig. 1 (a) The image of the specimen assembly. (b) The temperature dependence of resistance $R(T)$ measured at $160 \mathrm{GPa}$ for sample A. The multiple steps in the transition region imply there might be phases with different hydrogen amount in the synthesized calcium superhydrides. The inset is the enlarged view of the derivative of resistance over temperature $(\mathrm{d} R / \mathrm{dT})$ where the $\sim 210 \mathrm{~K} \mathrm{Tc}{ }^{\text {onset }}$ is determined as the upturn point at the right side.

Fig. 2 The resistance as a function of temperature measured at different magnetic field for sample A. The arrows are attributed to the phases with different hydrogen amount in the calcium superhydrides. The inset is the enlarged view of the resistance curves around the transition region of the $210 \mathrm{~K}$ phase.

Fig. 3 (a) The temperature dependence of resistance measured for samples B and C at $185 \mathrm{GPa}$ showing one step transition. (b) Resistance curves measured under different magnetic field for sample $\mathrm{C}$.

Fig. 4 The Ginzburg Landau fitting for the $H_{\mathrm{c} 2}(\mathrm{~T})$ shown with the solid lines. The stars represent the $H_{\mathrm{c} 2}(0)$ values calculated via WHH model. The inset of Fig. 4 is the critical field $\mathrm{Hc} 2(\mathrm{~T})$ as a function of $\mathrm{Tc}$ with the Tc values determined by the criteria of the $\mathrm{Tc}^{\text {onset }}, 90 \%$ and $50 \%$ of normal state resistance, respectively. 
Fig. 1
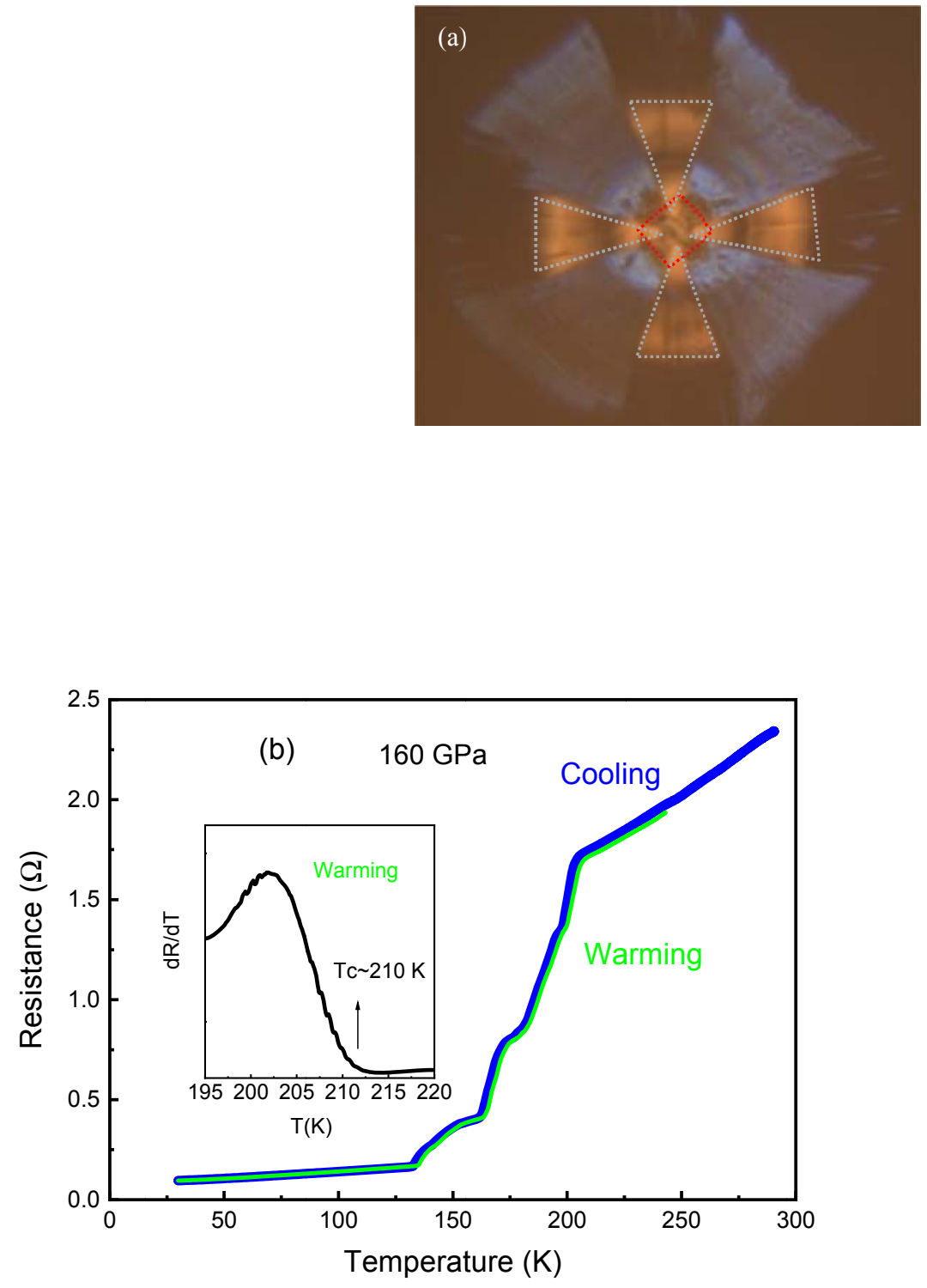
Fig. 2

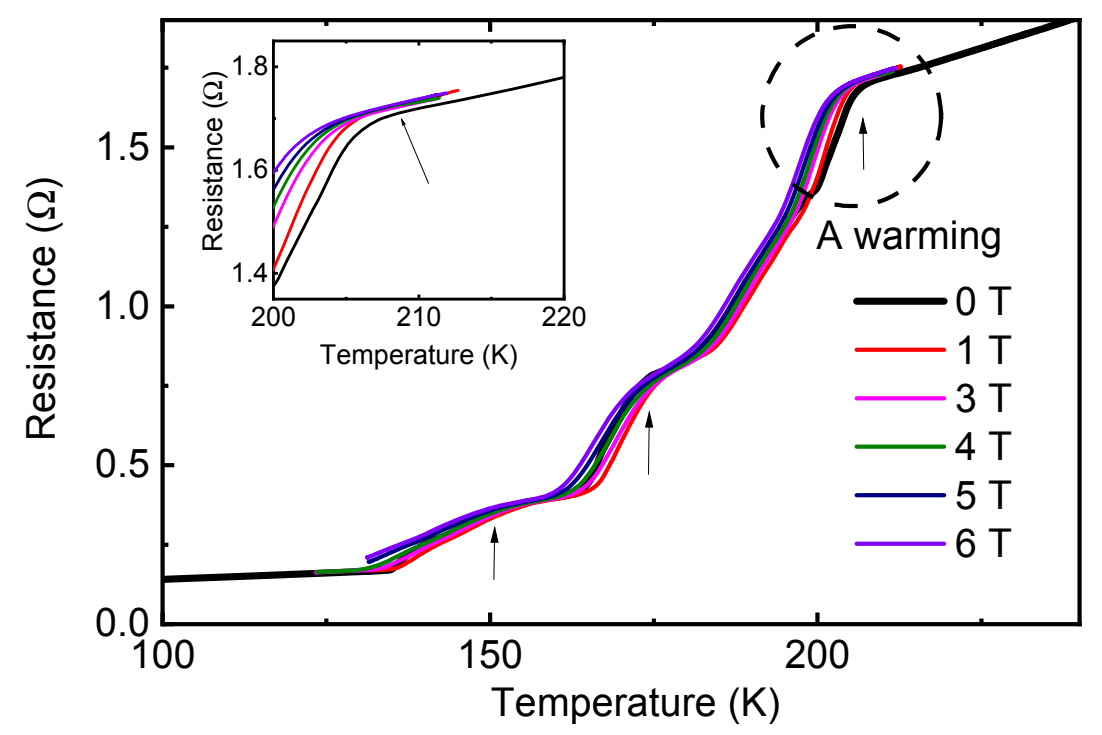


Fig. 3
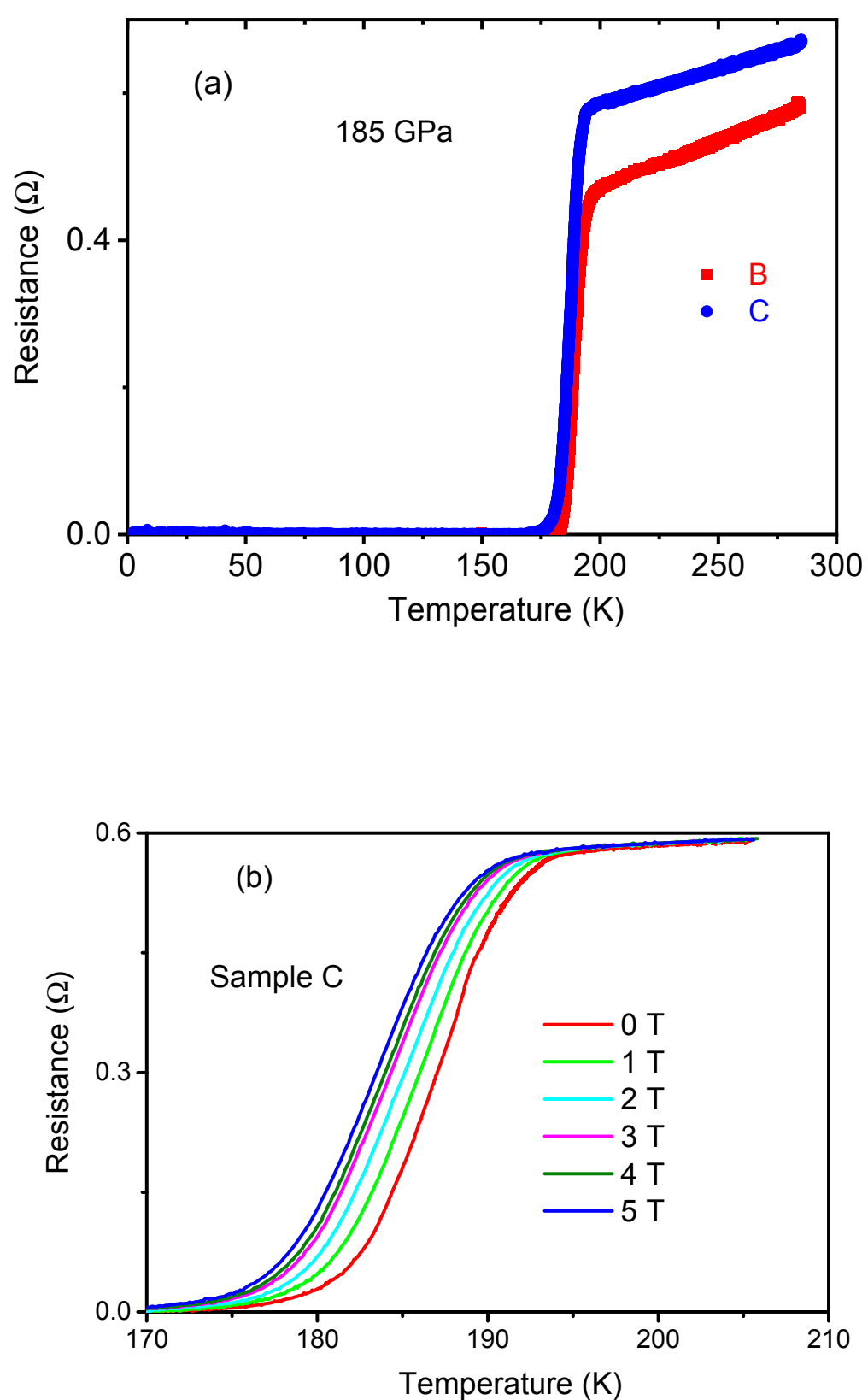
Fig. 4

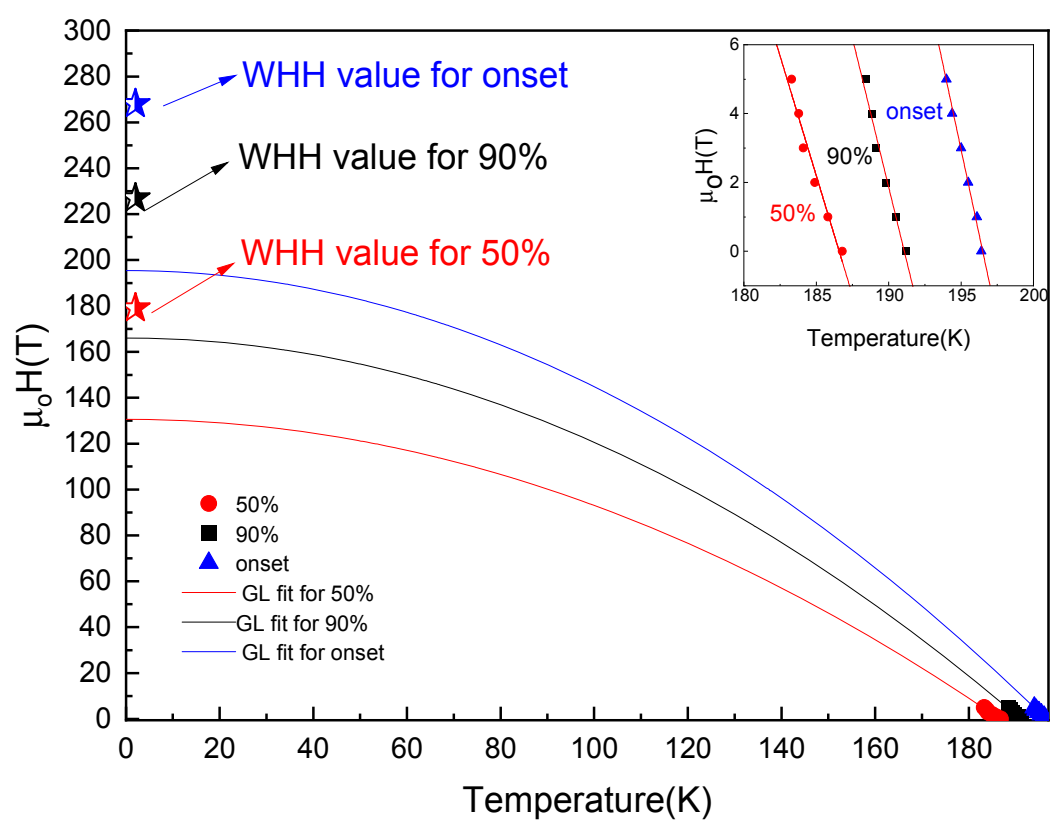




\title{
Supplementary materials
}

\section{Superconductivity above $200 \mathrm{~K}$ Observed in Superhydrides of Calcium}

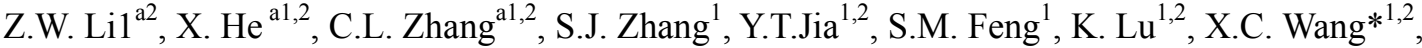 \\ J.F.Zhao ${ }^{1}$, Y. W. Long*1, ${ }^{1,2,3}$, R.C. Yu ${ }^{1,2}$, L.H. Wang ${ }^{4}$, M.Y. Ye ${ }^{5}$, Z.S. Zhang ${ }^{5}$, V. B. Prakapenka ${ }^{6}$, S. \\ Chariton $^{6}$, H.Z. Liu ${ }^{7}$, C.Q. Jin*1,2,3 \\ ${ }^{1}$ Beijing National Laboratory for Condensed Matter Physics, Institute of Physics, Chinese \\ Academy of Sciences, Beijing 100190, China \\ ${ }^{2}$ School of Physical Sciences, University of Chinese Academy of Sciences, Beijing 100190, China \\ ${ }^{3}$ Songshan Lake Materials Laboratory, Dongguan 523808, China \\ ${ }^{4}$ Harbin Institute of Technology, Harbin 130001, China \\ ${ }^{5}$ Jilin University, Changchun, China \\ ${ }^{6}$ Center for Advanced Radiations Sources, University of Chicago, Chicago, Illinois 60637, USA \\ ${ }^{7}$ Center for High Pressure Science \& Technology Advanced Research, Beijing 100094, China
}

Five strong peaks are observed, which of three can be indexed with lattice 
parameters of $a=b=c=3.3497 \AA$ and the systematic absence of $h k l$ implies the possible space group of $\operatorname{Im} \overline{3} m$; these results agree with the predicted for $\mathrm{CaH}_{6}$ at $150 \mathrm{GPa}$ $(a=b=c=3.501 \AA)$. Thus, we use the crystal structure of $\operatorname{Im} \overline{3} m-\mathrm{CaH}_{6}$ as the initial model to carry out the refinement for our diffraction data. After the other two strong unknown peaks marked by stars were excluded, the refinement converged smoothly to $R_{w p}=4.3 \%$ and $R_{p}=3.9 \%$. It is suggested that the main phase is possibly $\mathrm{CaH}_{6}$, which might respond to the superconducting transition observed with Tc exceeding $200 \mathrm{~K}$. However, if we compare the X-ray diffraction data with the theoretically predicted model of $\mathrm{C} 2 / \mathrm{m}$ phase of $\mathrm{CaH}_{12}$ under $150 \mathrm{GPa}$, it is found that the main peaks also seem to match the predicted peaks shown with the dark yellow line. In addition, for the sample $\mathrm{D}$, it shows superconducting transition at $189 \mathrm{~K}$ and $185 \mathrm{GPa}$ as shown in Fig. S3, which is lower than the calculated $\mathrm{Tc}$ of $\mathrm{CaH}_{6}$ but comparable with the Tc value predicted from $\mathrm{CaH}_{12}$. Therefore, we cannot rule out the possibility that the high temperature superconductivity found in our sample arises from the other calcium hydrogen phases.

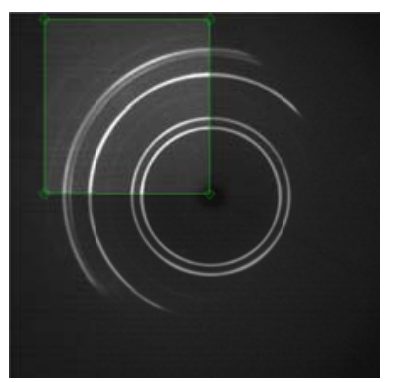

Fig. S2.1. The diffraction image plate with diffraction rinks.

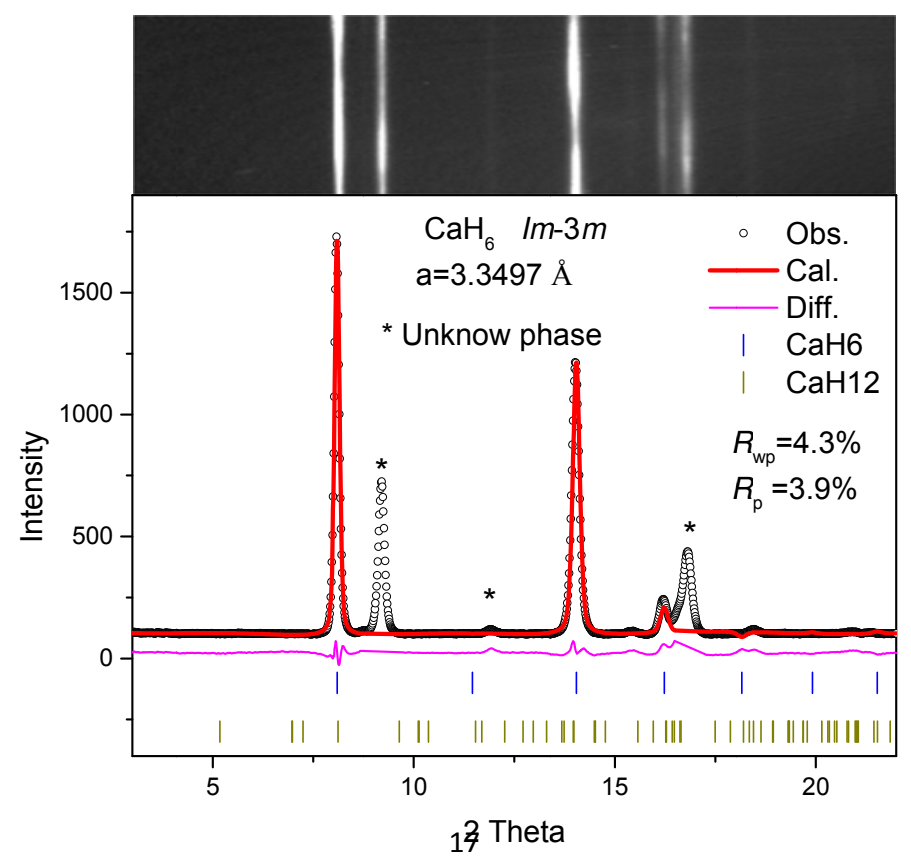


Fig. S2.2. The upper is the image along the azimuthal lines of the diffraction image. The main panel presents the diffraction pattern with integrated area shown with the square in Fig. S2.1.

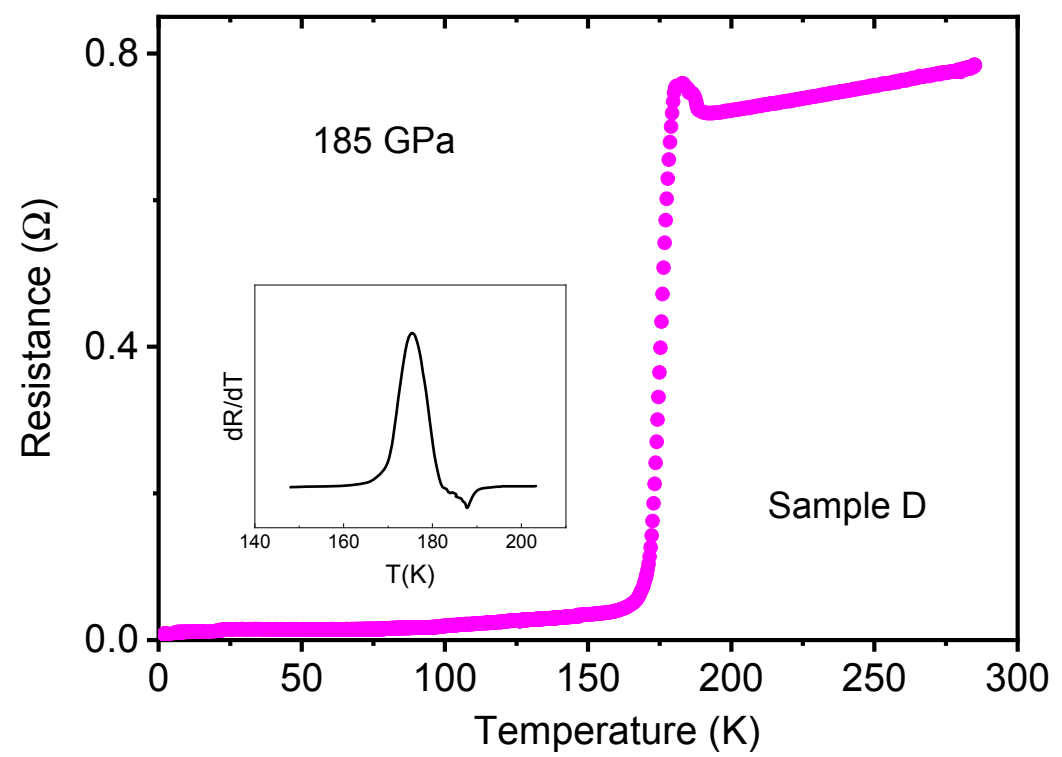

Fig. S3. The temperature dependence of resistance measured for sample D at 185 $\mathrm{GPa}$. The inset is the enlarged view of the derivative of resistance over temperature $(\mathrm{d} R / \mathrm{dT})$. 